\title{
Decentralized Trusted Computing Base for Blockchain Infrastructure Security
}

\author{
Thomas Hardjono ${ }^{1 * \dagger}$ and Ned Smith ${ }^{2 \dagger}$ \\ ${ }^{1}$ MIT Connection Science \& Engineering, Cambridge, MA, United States, ${ }^{2}$ Intel Corporation, Hilsboro, OR, United States
}

There is a growing interest today in blockchain technology as a possible foundation for the future global financial ecosystem. However, in order for this future financial ecosystem to be truly global, with a high degree of interoperability and stability, several challenges need to be addressed related to infrastructure security. One key aspect concerns the security and survivability of the systems that participate in the blockchain peer-to-peer networks. In this paper we discuss the notion of the decentralized trusted computing base as an extension of the TCB concept in trusted computing. We explore how a decentralized TCB

OPEN ACCESS

Edited by:

Pinar Emirdag,

State Street Corporation,

United States

Reviewed by:

James Burnie,

Eversheds Sutherland (International)

LLP, United Kingdom

David Challener,

Johns Hopkins University,

United States

${ }^{*}$ Correspondence:

Thomas Hardjono

hardjono@mit.edu

tThese authors have contributed equally to this work

Specialty section: This article was submitted to

Financial Blockchain,

a section of the journal

Frontiers in Blockchain

Received: 27 March 2019 Accepted: 25 November 2019 Published: 06 December 2019

Citation:

Hardjono T and Smith N (2019) Decentralized Trusted Computing Base for Blockchain Infrastructure Security. Front. Blockchain 2:24. doi: 10.3389/fbloc.2019.00024 can be useful to (i) harden individual nodes and systems in the blockchain infrastructure, and (ii) be the basis for secure group-oriented computations within the P2P network of nodes. We explore the application of the decentralized TCB for blockchain interoperability via blockchain gateways. Similar to border gateways in classical IP routing, blockchain gateways may provide several potential benefits in use-cases involving the transferal of virtual assets across different blockchain autonomous systems.

Keywords: trusted computing base, blockchain technology, infrastructure security, cryptography, survivability

\section{INTRODUCTION}

There is a growing interest today in blockchain technology as a possible foundation for the future global financial ecosystem. Significant media attention has been placed this new field, and various future visions for the "blockchain economy" has been put forward by various authors. The Bitcoin system proposed by Nakamoto (2008) is now over 10 years old, and the BTC currency remains one of the most popular speculative crypto-currencies.

One revolutionary aspect of the Bitcoin system is its openness for any entity to participate in the act of "mining." Any entity can independently and anonymously participate in the Bitcoin network by computing the "proof of work" (PoW) as part of establishing consensus regarding the state of shared ledger. As such, the membership in the Bitcoin network is not defined by geographic location. This in itself is a radical departure from traditional enterprise IP networks consisting of all known and authenticated entities. This independence and anonymity of the mining nodes means that it is difficult or even impossible to know which nodes participated in a given proof of work computation instance. This in turn leads to the possibility of anonymous mining pools amassing $\mathrm{CPU}$ power (hash power), which can be unleashed at the opportune moment in order to influence the network.

If the global financial industry is to employ blockchain technology for their future infrastructures, then several deficiencies of the current blockchain systems paradigm need to be addressed before blockchains can be used as a fundamental building block for financial technology. We believe that a crucial aspect of any financial IT infrastructure in their resiliency and survivability 
in the face of possible cyber-attacks. A core requirement for survivability is the interoperability of blockchain systems both at the mechanical (technical) level and at the value (economic) level. In fact, in the design of the architecture of the Internet the DARPA view at the time was that there are seven goals of the Internet architecture, with the first three being fundamental to the design, and the remaining four being second level goals. The first three goals were Internet communications survivability, the support for a variety of services types, and the support for a variety of networks (see Cerf and Khan, 1974; Clark, 1988). Therefore, chief among these goals was survivability, and in order to create survivable networks there must be a high degree of interoperability across these networks (see Hardjono et al., 2019).

In this paper we explore how the decentralized trusted computing base (DTCB) model can be useful to (i) harden individual nodes and systems in the blockchain infrastructure, (ii) to support cross-blockchain interoperability, and (iii) to enable secure group-oriented computations and interactions within blockchain networks. One underlying motivation for exploring the notion of the DTCB is to see if trustworthy interoperability can be achieved using the constructs of trusted computing. Thus, in this paper we also explore the idea of trustworthy interoperability using blockchain gateways that maybe implemented following the DTCB model.

In the next section we briefly review a number of challenges facing the nascent area of blockchain technology. In section 3 we review the history of the notion of the trusted computing base that emerged in the 1980s from the DoD Orange Book. We propose a number of desirable features of the DTCB in section 4 , building on the existing industry experience in trusted computing. Similar to other infrastructure technologies that have moved to the cloud, parts of the blockchain infrastructure may also end up moving to the cloud environment. We discuss the role of the TCB in the context of virtualized and cloud environments in section 5, paying close attention how the roots of trust can be extended into these virtualized containers. In section 6 we discuss an important use-case of the DTCB related to the interoperability and survivability of blockchain infrastructure, namely the construction of a secure gateway that interconnects blockchain systems. We close the paper with some future considerations.

\section{CHALLENGES IN BLOCKCHAIN SYSTEMS}

The emergence of the Bitcoin system has provided the first working example of a "permissionless" blockchain system pertaining to digital currency. The term "permissionless" and "trustless" today is used typically to convey the fact that anyone (end-users and mining nodes) are free to join or leave the P2P network at any time. As such, the "membership" of a permissionless blockchain system is dynamic and the perimeter is elastic.

However, there are a number of limitations to the permissionless blockchain design:

- Anonymity of nodes may lead to concentrations of hash-power: In some permissionless blockchain networks, any entity can take the role of a mining node and be identified on the blockchain solely by their public-key (i.e., "address"). Although this anonymity may be considered as a virtue in some blockchain networks (e.g., Bitcoin), there may be some disadvantages to this approach. One disadvantage is the potential for the amassing (centralization) of hashing power by a handful of anonymous nodes or entities, which goes against the proposition of decentralization of the blockchain paradigm. Such entities could conceivably use this concentration of hash-power to skew or manipulate the network over time.

- The trustless model may only achieve limited trust: The trustless model based on anonymous nodes independently completing the proof-of-work achieves only limited trust (technical-trust). The lack of measurable security quality or of strong proofs of security may have a negative impact of the ability to forecast the reliability of services built atop the trustless network of nodes. This may in turn affect the ability to establish service level agreements or contracts.

- Limitations in technical-trust impacts business-trust: The limitations in measurable technical-trust and security quality inherent in the trustless model as employed in some blockchains impacts business in the sense that businesses are unable to count on the availability, resilience or performance of a given trustless blockchain. This is in contrast to the Internet today, which is composed of a set of ISPs (e.g., local, metro, and backbone), and which operate based on peering agreements and SLAs. Without service agreements or contracts, there is only a very limited amount of risk that businesses are willing to take on (see Lipton et al., 2018).

Currently blockchain systems follow one of the following general approaches to the membership of nodes:

- Pre-identified participation: Nodes must be identified and authenticated prior to joining the community. This does not imply, however, that a node will participate in every instance of a consensus computation. Examples of these are some deployments of Hyperledger Fabric (see Androulaki et al., 2018).

- Anonymous participation: In this approach the nodes are not identified or authenticated as a member of the community. Thus, any entity with computing resources can operate a node within the community, and the node can "come and go" as it pleases. This approach is exemplified by the Bitcoin system.

- Anonymous-verifiable participation (hybrid): In this approach a node is able to cryptographically prove it is a member of a blockchain system without revealing its full identity (for example, see the scheme proposed in Hardjono and Smith, 2016 based on the EPID standard ISO/IEC, 2013).

\section{THE TCB MODEL: A SHORT HISTORY OF TRUSTED COMPUTING}

\subsection{Orange Book Trust}

In December of 1985 the U.S. Department of Defense published the Trusted Computer System Evaluation Criteria (TCSEC) that defined Trusted Computing Base (TCB) as "the security-relevant 
portions of a system.” All subsequent expressions of trustworthy computing and security policy is described in terms of impact and relevance to the TCB. The TCSEC notion of TCB is most easily understood if it behaves as a centralized trusted entity. Indeed, those systems that achieve the highest-level security certification and accreditation have a very centralized system design. Security Enhanced Linux (SELinux ${ }^{1}$ ) embodies this thinking in its design for Linux Security Modules (LSM) where security relevant operating system events must satisfy LSM imposed security policies. This approach is made feasible largely due to the Linux architecture that regards everything in the system as either a subject or an object. Subjects operate on objects and objects expose resources. The LSM sits at the confluence of subjectobject interaction mandated by kernel system calls. Using TCSEC conventions, a Linux LSM is the primary TCB component of the operating system.

The TCSEC embraces the notion of peer trusted nodes describing them as members of a... "Network Trusted Computing Base (NTCB) [which] is the totality of protection mechanisms within a network system-including hardware, firmware, and software-the combination of which is responsible for enforcing a security policy [...] An NTCB Partition is the totality of mechanisms within a single network subsystem...". The TCSEC NTCB criteria, however, does not include the concept of attestation where trustworthiness properties can be automated and dynamically evaluated.

\subsection{The Trusted Computing Group}

The TCSEC criteria focused mostly on operating system security. However, the operating system is not the sole TCB component in a platform. The hardware also plays a significant role as memory page isolation is central to the idea of kernel-mode (namely ring0 ) and application-mode (namely ring-3) security context. From the operating system perspective the hardware is trusted because it has no alternative way to test and verify that the hardware is behaving correctly. This does not mean that hardware is not susceptible to compromise, as indicated by recent exploits such as Spectre ${ }^{2}$ and Meltdown ${ }^{3}$.

The threat of hardware vulnerability motivated the computing industry to form the Trusted Computing Group ${ }^{4}$ (TCG) where the notion of a hardware root-of-trust was used to distinguish the security relevant portions of a hardware platform. The TCG defines trusted computing ${ }^{5}$ more organically building upon granular components that are described as shielded locations and protected capabilities. Shielded locations are "A place (memory, register, etc.) where it is safe to operate on sensitive data; data locations that can be accessed only by protected capabilities." Protected capabilities are "The set of commands with exclusive permission to access shielded locations."

Using these concepts, the TCG breaks down trusted hardware functionality into three components, otherwise referred to as "roots-of-trust." There is a Root-of-trust for Measurement (RTM)

\footnotetext{
${ }^{1}$ https://github.com/SELinuxProject

${ }^{2}$ https://spectreattack.com/spectre.pdf

${ }^{3}$ https://meltdownattack.com/meltdown.pdf

${ }^{4}$ https://trustedcomputinggroup.org

${ }^{5}$ https://trustedcomputinggroup.org/resource-directory/glossary/
}

whose primary role is to ensure the rest of the platform initializes and boots correctly. The Root-of-trust for Storage (RTS) ensures saved security relevant state, cryptography objects (e.g., keys) are persistently available for inspection and use regardless of whether the surrounding platform firmware and software has been compromised. The third root is called Root-of-trust for Reporting (RTR) that contains cryptographic algorithms, protocols and access to RTS protected keys so that peer nodes can assess trustworthiness properties dynamically. This assessment is referred to as attestation.

\subsection{The Trusted Platform Module}

According to the TCG, a Trusted Platform Module (TPM) is formed by combining an RTS and RTR, but the TPM specification does not clearly delineate which features are ascribed to RTS vs. RTR. Generally speaking, a TPM provides four security capabilities:

1. Cryptographic libraries including a true random number generator (TRNG), key generations and key storage;

2. Platform Configuration Registers (PCR) containing firmware and software digest values supplied by an RTM or other code loaded by the RTM. The RTM is supposed to be securely connected to the TPM;

3. Sealing and binding where data encrypted using TPM keys are locked to the platform containing the TPM. Sealing expects the PCRs will have a prescribed value as an additional constraint upon decryption;

4. Remote attestation uses TPM keys to sign PCR values that are delivered to a remote entity for evaluation. Attestation keys are protected by the TPM; which contains an endorsement key that is provisioned at TPM manufacturing and certified by the manufacturer's certificate authority (CA). The endorsement certificate establishes TPM provenance and a platform attribute certificate issued by the platform OEM establishes platform provenance.

The first successful version was TPMv1.1b followed by TPMv1.2 which was widely distributed. TPMv1.2 supported a "one-sizefits-all" approach that primarily targeted the PC market. It defined a single storage hierarchy protected using a single storage root key (SRK). Authorization could be asserted using an HMAC, PCR value, locality, or assertion of physical presence. It supported unstructured Non-Volatile RAM storage and a variety of cryptographic algorithms including SHA-1, RSA, AES, 3DES, MGF1 mask generation, and Direct Anonymous Attestation (DAA), added to TPMv1.2, is a zero-knowledge algorithm. It mandated 24 PCRs that use SHA-1 hash computations.

A second generation TPM v2.0 was a library specification and is not backward compatible with TPMv1.2. It expanded trusted computing features to better support vertical markets. The TCG introduced platform specific profiles that were designed to use optional or excluded functionality specific to PCs, smart phones and automotive IoT platforms. Platform-specific profiles allow TPM vendors flexibility in implementing TPM features that accommodates a specific market. Additionally, TPMv2.0 supports four key hierarchies; storage, platform, and endorsement as well as a hierarchy for ephemeral keys. 
Each hierarchy can support multiple keys and cryptographic algorithms. Password-based authorization was added and greater flexibility for policy-controlled use of the other authorization mechanisms. NV-RAM expanded to support monotonic counters, bitmaps and "extend" operations in addition to unstructured data storage. Support for stronger cryptographic algorithms was added including SHA256 for hashing and ECC using Barreto-Naehrig 256-bit curve and NIST P-256 curve. The 128-bit AES key size became mandatory. TPMv2.0 also expanded the number of hash algorithms used to compute PCR values. For example, both SHA-1 and SHA256 hash values can accompany the same PCR register for added security and interoperability.

\subsection{Intel SGX Root of Trust}

The Intel Software Guard Extensions (SGX) (see Mckeen et al., 2013) offers another perspective on trusted computing base where a trusted environment exists within a user process called an Enclave. The SGX TCB consists of hardware isolated memory pages, CPU instructions for creating, extending, initializing, entering, exiting, and attesting the enclave and privileged $\mathrm{CPU}$ modes for controlling access to enclave memory. SGX takes a layered approach to TCG design where CPU hardware and microcode make up the bottom layer consisting of Enclave Page Cache (EPC), EPC Map (EPCM), and protected mode operation logic. We refer to this as the CPU TCB.

A second layer TCB consists of SGX runtime code that includes a user or ISV supplied SGX runtime. We refer to this as the ISV TCB. Finally, enclave runtimes may dynamically load third layer code and configuration data that further specializes enclave behavior. We refer to this as the application APP TCB. Intuitively, from the application's point of view, application functionality within the enclave is the subset that the application developer designates as trusted. For example, it may contain sensitive application data, algorithms and cryptographic keys off limits to other processes and enclaves.

The three TCB components (CPU TCB, ISV TCB, APP $\mathrm{TCBt}$ ), together, make up the subset of all processes that are trusted, collectively known as the PLATFORM TCB. There is a trust dependency within the PLATFORM TCB, as APP TCB functionality must trust ISV TCB and ISV TCB must trust CPU TCB. However, in the broader context, all SGX TCB elements do not need to trust external ring-3, ring-0, and VMX root functionality.

SGX architecture supports helper enclaves known as Architectural Enclaves that perform various trusted computing services common to most all application enclaves. These service enclaves include: the Platform Configuration Enclave (PCE) that facilitates provisioning certificates for identity and attestation; the Quoting Enclave (QE) for performing enclave-to-enclave attestation as well as remote attestation and the Platform Services Enclave (PSE) for interacting with IP blocks that exist outside the CPU IP complex. Application enclaves may include and exclude architectural enclaves as needed to satisfy application objectives. In this sense, the PLATFORM TCB is dynamic, at least from the application developer's perspective.
A second generation SGX (see McKeen et al., 2016) added support for dynamic memory management where enclave runtimes could dynamically increase or decrease the number of enclave pages. This is a second form of TCB dynamism where if we consider the identity of a TCB to be a function of the code or logic executing within the TCB (e.g., cryptographic hash), dynamically loaded or unloaded pages also changes the TCB identity. Trust decisions that anticipate a particular TCB could become confused by dynamic memory management. There are at least two ways to address this. One approach requires all the pages that could be dynamically loaded be included in the hash computation then unused pages can be evicted when not needed. Alternatively, a TCB identity not tied to a hash function is used. For example, the vendor could assign a product name that is verifiable using a certificate. This approach gives vendors greater flexibility toward code maintenance, but at the cost of potentially introducing new vulnerabilities. SGX allows both TCB naming approaches but with one important augmentation. Each TCB element has a Security Version Number (SVN) that the TCB vendor uses to track security relevant changes. SVN is a monotonic value designed to detect and, in many cases, can be used to prevent version regressions. It requires a level of discipline on the vendor's behalf to ensure SVN is managed correctly. An important benefit of SVN is it reduces the number of unnecessary TCB re-certifications due to code maintenance activities having no security consequence.

\section{DESIRABLE PROPERTIES OF A DECENTRALIZED TCB}

There are a number of core properties desirable for a Decentralized TCB (DTCB) model. Individually, each participating node in a DTCB instance must possess the fundamental properties of trusted computing, and more specifically the core aspects of trustworthiness (technical trust). In this context it is useful to revisit some key architectural designs of the Trusted Platform Module (TPM) (see Trusted Computing Group, 2003b) established by the TCG from the late 1990s which sought to embody (implement) technical trust in hardware.

\subsection{Properties for Technical Trust}

We say that a node (i.e., system composed of hardware and software) can be considered to exhibit technical trust if at least the following properties apply:

- TCB Property P1: Performs a well-defined function. The core idea here is that the function being executed by the TCB must not harm the TCB itself and must be computationally bounded so that it does not consume all available resources. In the TPM1.2 design, these functions consisted of a set of primitive operations and some cryptographic functions. In recent systems such as SGX, there is more freedom for the user to load arbitrary functions into the trusted execution environment to be executed, but the property remains true, namely that a user-defined routine must execute within the memory space determined by the SGX systems itself. 


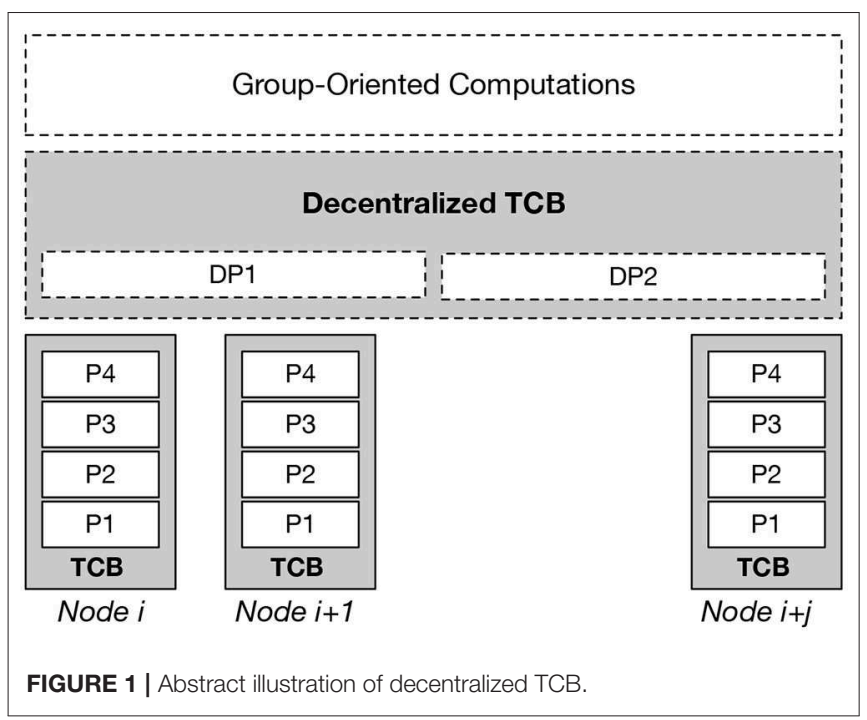

- TCB Property P2: Operates unhindered and shielded from external interference. In order for an implementation of a function to be useful in a TCB, the function must be able to execute until its completion without being hindered in any way (e.g., resources locked or made unavailable) or that its operations are not skewed or influenced in any fashion.

- TCB Property P3: Cryptographic Identity. TCB instances must be distinguishable from each other. Trust associations between application layers and TCB nodes will differ in deployment; unless mirrored for fault-tolerance. Cryptographic identity ensures each TCB node possesses a unique identity and can prove that identity to a peer node.

- TCB Property P4: Trustworthy TCB Dynamism. For a TCB to be practical, it must be able to be updated, expand, and contract. Few instances of code are $100 \%$ error free. Those that are proven to be error free are highly constrained making them nearly impractical for interesting use cases. The most relevant use cases involve establishment of a cryptographic identity and firmware or microcode update. Beyond these use cases the TCB needs to be dynamic allowing layers of functionality to be added (or removed) to account for changing hardware and workload computational requirements. The authors note that TCB dynamism is antithetical to a design goal which seeks to make roots of trust immutable. Often it is necessary to simplify (i.e., remove features) in order to achieve a higher degree of immutability. Simpler roots of trust implies there will be TCB layers that add the necessary functionality back in. The TPM is a fairly complex root of trust and not surprisingly needed an interface for updating it in the field.

We can further say that a node effectively participates in forming a DTCB if at least the following properties apply (see Figure 1):

- DTCB Property DP1: Group Membership. A DTCB consists of a group of TCB nodes where group membership criteria are applied. Membership enforcement ensures a non-TCB node does not become a member and compromised or non-compliant TCB nodes are expelled from the group.
For DTCB establishment, the group of TCB nodes must cooperatively verify potential DTCB member nodes are authorized as members. The authors point to distributed consensus algorithms as a possible approach for group membership enforcement.

- DTCB Property DP2: Truthful Attestation. For a DTCB to be practical, it must be able to truthfully report the result of its static and dynamic composition, internal execution or function and status of its resources (e.g., registers, memory usage, etc.). Although the industry has defined several attestation solutions (e.g., FIDO key attestation, see Jones, 2015; Android key attestation, see Android, 2019; Microsoft TPM key attestation, see Microsoft, 2017; Intel SGX, see Anati et al., 2013), they do not consider its role in establishing a DTCB. For DTCB establishment, the group of TCB nodes must cooperatively verify potential DTCB member nodes' TCB trust properties are aligned with vetted DTCB policies.

\subsection{Possible Group-Oriented Features}

Based on the above three technical-trust properties, there are a number of possible features that maybe achieved using the above properties:

- Anonymous Group Membership: Following from property DP1 above, the use of hardware to implement the three technicaltrust properties allows a node to employ (reveal) different degrees of device-identification and ownership-identification, depending on the type of blockchain network it is participating in. For example, in the case of permissionless networks where the anonymity of nodes is desirable, a node may use verifiable anonymous identities (see Camenisch and Van Herreweghen, 2002; Hardjono and Smith, 2016) to prove it is a legitimate member of the network without revealing which member it is.

In strongly permissioned blockchain systems, a node could reveal the manufacturing details of all its hardware, firmware, and software to an authorized verifier in order to prove that the node complies to the minimal operational requirement of the permissioned blockchain infrastructure.

- Group Reporting: Following from property DP1 above, a group of DTCB nodes can employ a group-oriented quote protocol as a counterpart of the single hardware quote protocol (see Trusted Computing Group, 2003a). Among others, the group-quote protocol could require each participating node to perform some internal secret computation, and report its result using the traditional quote protocol to other members of the group. A group-shared secret key could additionally be deployed to protect the transmission of each quote result.

Another more general use of group-reporting is for a group of nodes to report (to each other) their respective system manifests, namely a cryptographically signed list of its hardware, firmware and software installed (for example, see the TCG signed manifest in Hardjono and Smith, 2006). This is crucial in cases where nodes are operating different versions of hardware and software (e.g., version of mining software), and where a given version of a software may be known to 
possess bugs and/or susceptible to malware attacks which may dramatically impact the blockchain as a whole.

- Group Computation Participation: The ability for a node to truthfully report (prove) its internal hardware status (e.g., registers) allows the nodes to prove that it actively participated a given group-consensus computation.

New consensus computation algorithms could be designed which would embed anonymous-verifiable identification of a given node as a precondition for the successful outcome of that node's proof-of-work (PoW). Similarly, a confirmation broadcasted by a node could require that the node also anonymously attach proof of its DTCB-capabilities.

In the next section we discuss the use of DTCBs in virtualized cloud environments, motivated by the fact that parts of the blockchain infrastructure (e.g., mining nodes) may operate in a hosted environment-which faces a number of security challenges in itself.

\section{HARDWARE ROOTED TCBS IN VIRTUALIZED CLOUD ENVIRONMENTS}

This section describes common hosting environments for containers, another form of distributed system popular among cloud and network edge service provider networks. It further illustrates techniques for dynamic establishment of ТCB cryptographic identities and TCB layering. When correctly applied, container environments can be formed with a hardware rooted TCB otherwise known as a hardware root of trust (RoT). Hardware RoT is an essential ingredient in the formation of a decentralized TCB.

A few typical deployment models consist of decentralized compute (aka containers) with centralized orchestration (Figure 2). A more sophisticated model has decentralized, but federated orchestration with decentralized pools of containers (Figure 3). Still a third model employs Function-asa-Service (FaaS) to decompose workloads into their functional components, where function execution is distributed across multiple function provider nodes. Application execution and workflow logic encapsulates "orchestration" resulting in decentralized orchestration with decentralized function execution (Figure 4).

Multi-tenancy is a security challenge facing all deployment models. Client workloads are presumed to be mutually suspicious and therefore at risk for digital espionage from among the tenant community. PaaS, FaaS, and orchestration hosting environments are expected to provide appropriate tenant isolation to counter potential attacks.

Additionally, network-based attackers could pose man-inthe-middle threats that require end-to-end confidentiality and integrity protection of workload payloads as they propagate among the various stations. Multi-tenant isolation and end-toend cryptography technologies must be effectively used to ensure comprehensive security. Although these security capabilities alone are not enough to ensure reliable operations.

PaaS servers may advertise container pools having equivalent workload hosting capabilities, but they may indeed differ. Proof of algorithm equivalency is a desirable property when interchangeable function and workload hosting environments exist. Ideally, a scheduling routine should check function equivalence as a precondition of committing the execution resource.

Federated orchestration may require distributed consensus at the orchestration level. Workload scheduling involves management of task queues where tasks may complete early, late, or on time. Delivering task results incurs latency from server to client and may require temporary caching due to availability of both. Task statistics inform regarding resource utilization and performance optimization. They are also essential to SLA compliance, accounting, and billing processing. Decentralized ledger technology can be used to track task scheduling statistics so that servers accurately report resource utilizations countering potential accounting and billing fraud. Federated orchestrators can more conveniently avoid workload server oversubscriptions using blockchain to track task queue status.

Decentralized orchestration using Service Level Agreements (SLA) that describe compensation for performing the algorithm can prove all parties stake is resolved equitably according to the SLA contract. In a truly decentralized FaaS system, workload decomposition, and workload scheduling are themselves functions to be processed by the FaaS fabric. SLA compliance checking could involve collection, processing and verification of multiple auditing and accounting logs spread across dozens or even thousands of compute nodes in a FaaS fabric. Any one system failing to keep accurate logs potentially disrupts processing across the entire FaaS system. Blockchain proof of stake algorithms ensures audit and accounting logging is performed with redundancy and guards against isolated cheating. SLAs describing compensation for performing the task can prove all parties' stake is resolved equitably according to the SLA contracts.

Multi-tenancy, task isolation and end-to-end cryptographically protected workloads, SLAs, accounting, and telemetry data motivate application of trusted computing principles across Edge and Cloud computing infrastructures. The cryptographic identities of the various nodes and their roles need to resist impersonation attack. Cryptographic keys that protect the confidentiality and integrity of the data need to resist attacks while in flight and while cached or stored. Function executions need to resist attacks on code while it executes. To achieve these goals, it is essential that the nodes involved in the decentralized Edge and Cloud computing enterprise assess trustworthiness properties before placing data, code, and resources at risk. Attestation procedures applied when computing resources are placed into operation and periodically during use ensures expected operational integrity characteristics are in place as a pre-requisite to decentralized application executions. The DTCB is primarily tasked with establishment of trustworthiness pre-requisites.

\subsection{TCB Layering}

DTCB trustworthiness can be understood in terms of its roots-of-trust components and its methodology for TCB layering and update. The Trusted Computing Group has 


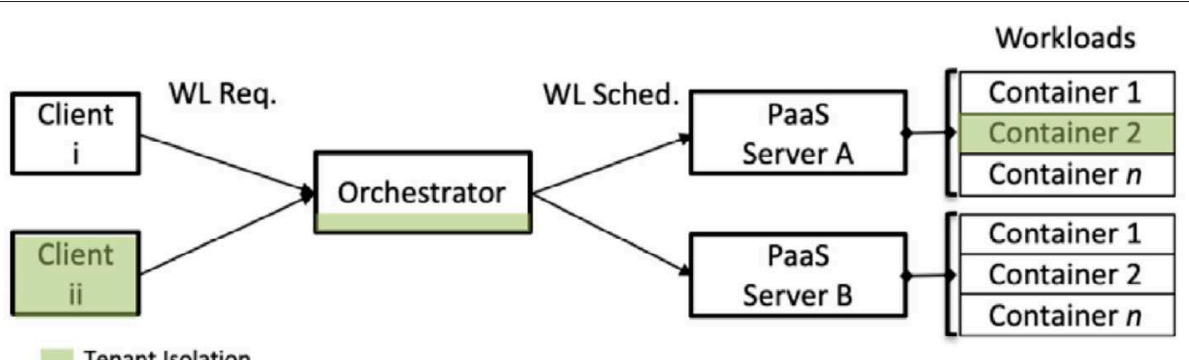

Tenant Isolation

FIGURE 2 | Centralized orchestration of container pools.

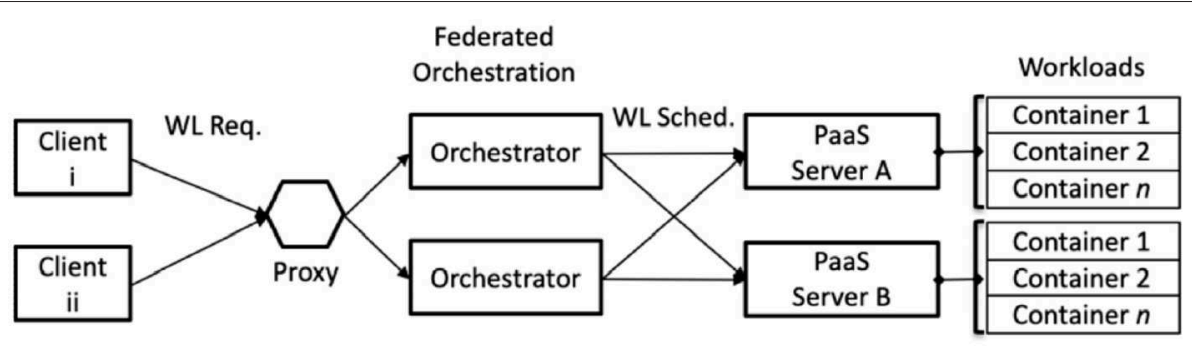

FIGURE 3 | Federated orchestration of container pools.

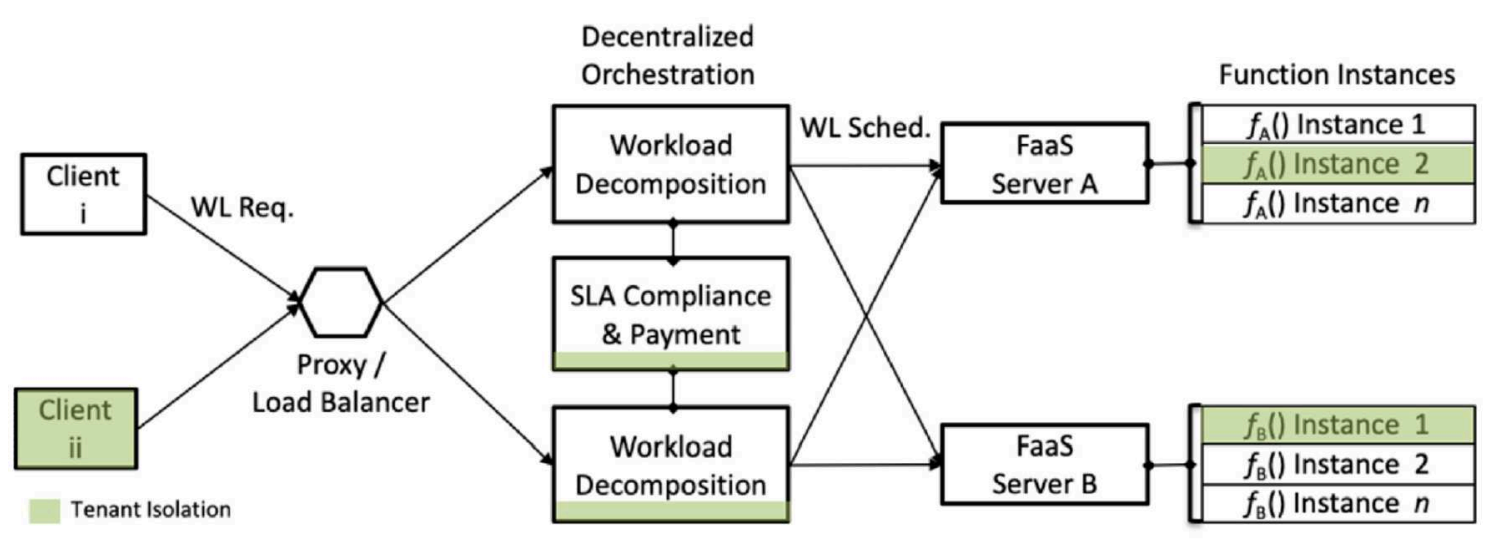

FIGURE 4 | Decentralized orchestration with decentralized FaaS.

defined Device Identity Composition Engine (DICE)see Trusted Computing Group, 2016-which is a trusted hardware building block for generating cryptographic device identities and attestation using the identities. The hardware implementing DICE is the first layer of a layered TCB architecture. Subsequent TCB layers can be dynamically added or removed to fallback to a trusted state. TCB layers may be added during manufacturing and later at or during deployment.

The Layered TCB (LTCB) approach seeks to identify the most essential trusted computing components implementable in hardware and whose implementation is verifiably correct. Techniques for dynamic TCB layering are also a consideration for LTCB design.
The following are some considerations for TCB layering:

- Hardware Root of Trust (ROT): The base layer capabilities are trusted and implemented in hardware. That is to say they are immutable or that mutability is highly constrained. For example, programmable integrated fuses may be set during manufacturing but remain immutable subsequently. Algorithms for computing cryptographic one-way functions, key derivation and key generation functions can have immutable hardware implementations. Circuit power-on and bootstrapping control logic can be immutable. Other TCB logic may be mutable but only under well-defined conditions. For example, a CPU micro-code may be patched post manufacture. 
- TCB Layer Identity: Subsequent layered TCB environment is unambiguously distinguishable. For example, the product ID of an Intel Core processor with virtualization identifies an environment where a CPU mode switch causes a hypervisor to execute in VMX Root. The microcode patch level or SVN further distinguishes the TCB layer. Many Intel CPUs have a monotonically increasing Security Version Number (SVN) that changes whenever a security relevant change is made to micro-code. The loaded hypervisor image also distinguishes the TCB layer. Collectively these attributes identify a hypervisor-based TCB. However, it does not disambiguate instances of the same TCB. It may be necessary to establish a cryptographic identity for a TCB layer for on-boarding, resource management, auditing, accounting, and telemetry.

- Inspect-ability of Next Layer Software: Because TCB identity can be a function of the software that is dynamically loaded, the current layer TCB must be able to inspect the next layer software. Inspection may simply be to compute a hash value for computation of a TCB identity or may involve more rigorous proofs of integrity and expected behavior.

- Layer Sequencing: Depending on the design of the hardware ROT, layering sequence may be relevant. For example, the current layer TCB may exclusively depend on the most recent layer for all of its trusted capabilities. Other architectures may allow subsequent TCB layers direct access to the hardware ROT.

- Layer Attestation: TCB layers may provide security functionality that is unique to a platform or system. These interactions patterns do not necessarily follow layer sequencing patters. Therefore, it may be appropriate to precede inter-layer interactions with layer attestations to establish a layer's trustworthiness profile. Layer attestation primitives also support construction of DTCB as will be discussed later.

\subsection{Examples of TCB Layering}

This section discusses several examples of TCB layering architectures. The first (Figure 5) highlights a DICE architecture consisting of hardware (Layer -1) containing two trusted capabilities: (i) the Unique Device Secret (UDS) and (ii) Compound Device Identifier (CDI) function.

The Unique Device Secret (UDS) is a one-time programmable globally unique value. Its only use is to seed a Compound Device Identifier (CDI) function that, combined with an First Mutable Code (FMC) value, generates a symmetric secret that is specific to the layer that provided the FMC. The FMC combined with Layer 0 product ID information identifies the Layer 0 TCB. The CDI function is a one-way function that uses the UDS to produce a keyed hash of the FMC called the CDI. The CDI uniquely identifies the Layer 0 TCB.

The CDI is securely installed into the Layer 0 environment where it serves two purposes: (i) to seed a one-way function for creating a Layer 1 symmetric secret, and (ii) to seed a device identity generation function. For example, $f()_{D E V I D}$ could be an RSA key generation function where CDI is used to seed its random number generator. The DeviceID $D_{L 0}$ is an asymmetric

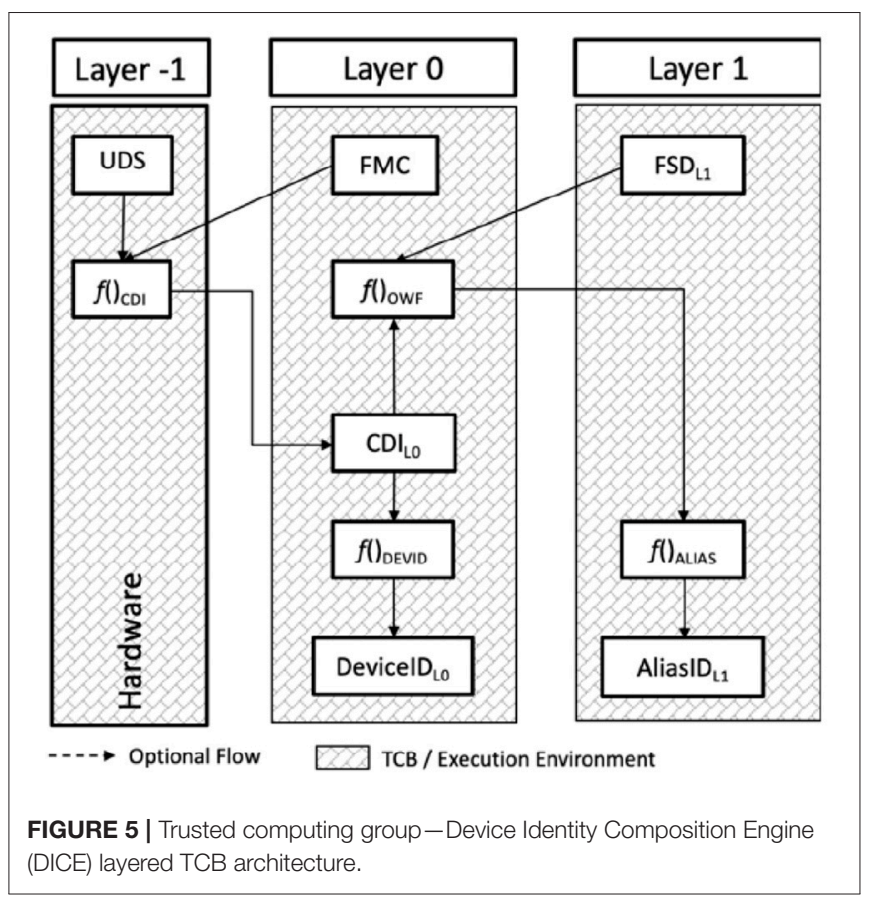

unique Layer 0 identifier that may be suitable for a variety of user defined deployment usages.

The Layer 1 TCB is identified using the Firmware Security Descriptor (FSD) which is the firmware hash component of the one-way function [i.e., $f()_{O W F}$ ] found in the Layer 0 TCB that computes the Layer 1 symmetric secret used to seed the $f()_{\text {ALIAS }}$ function that generates AliasI $D_{L 1}$ that uniquely identifies the Layer 1 TCB.

A generalization of the DICE architecture layering (Figure 6) can be inferred following the naming convention where the Layer 0 TCB identity is known as the Current TCB Context (CTC) and the Layer 0 unique TCB identity is known as the Previous TCB Context (PTC) because it captures Layer -1 TCB layering dependency. Although the hardware (Layer -1 ) TCB does not have a previous layer dependency, the UDS provides uniqueness. Optionally, the one-way function could accept a Layer -1 TCB identity value $C T C_{L-1}$ [though not described in the specification from the Trusted Computing Group (2016)].

Subsequent layers each rely on its respective previous TCB layer to provide a one-way function that inspects the current (to be instantiated) layer $C T C_{L_{n}}$ and the unique $P T C_{L_{n-1}}$ identifier to produce the current layer's unique identifier $P T C_{L_{n}}$. The PTC value propagates both the platform uniqueness property (inherited from the UDS), layer uniqueness (UDS + CTC) and layer sequence property; which is the combination of all prior $f()_{\text {OWF }}$ functions.

A third example (Figure 7) shows Intel SGX layered TCB architecture. In this architecture each layer has access to the Layer -1 TCB. The Layer -1 TCB is identified using the CPU product ID and CPU SVN values. A UDS provides platform uniqueness. The one-way function is always performed by a hardware RoT in Layer -1 . 


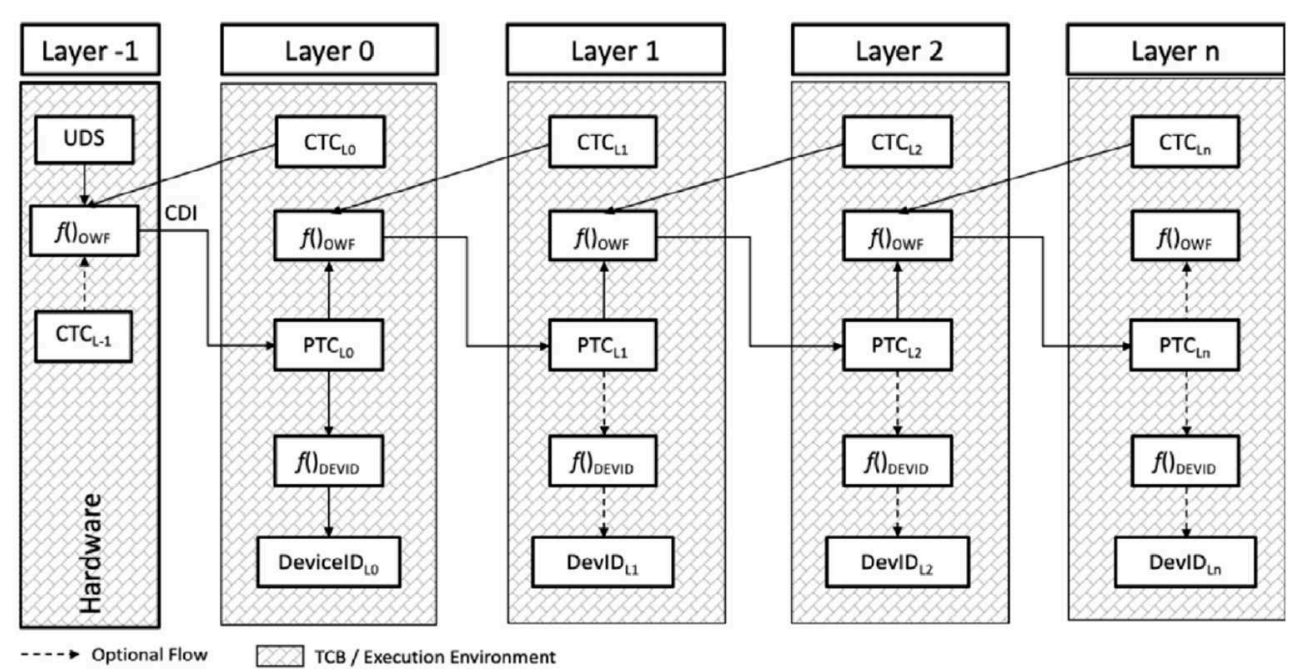

FIGURE 6 | A generalized Device Identity Composition Engine (DICE) layering architecture.

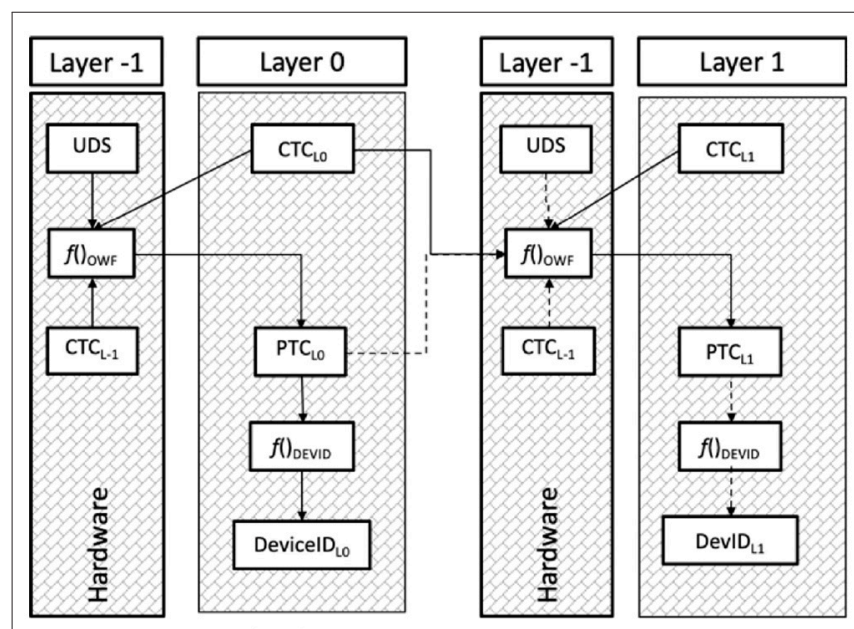

-..- Optional Flow $\square$ TCB / Execution Environment

FIGURE 7 | Intel SGX layered TCB architecture.

SGX layers have more flexibility (than the generalized DICE TCB layering) in that the PTC computation does not require inclusion of the Layer -1 UDS and CTC. However, the Layer -1 TCB supplies a local attestation capability that allows any enclave environment the ability to assess layering semantics. If layer sequence semantics are important to a user deployment, the expected layering can be created. Expected layering semantics can be verified using a combination of local and remote attestation.

Given a user deployment focus on trustworthy Edge and Cloud computing, use of trusted computing techniques that include hardware RoT and TCB layering play a vital role. Container environments may have widely varied trust properties. As Edge and Cloud ecosystems become more democratized and complex, reliance on distributed trust becomes essential.
Attestation is a mechanism whereby verifiers, presumable the entity fulfilling an SLA contract and the user community they represent, seek to manage risk associated with automation complexity and ecosystem diversity.

By incorporating principles of trusted computing into a decentralized TCB layer, many of the challenges facing application developers seeking predictable deployment in Edge and Cloud environments can be modularized for ubiquitous availability and relied upon for consistent trusted computing behavior.

\section{USE-CASE: GATEWAYS FOR BLOCKCHAIN INTEROPERABILITY}

Given the history of the development of the Internet and of computer networks in general (e.g., LANs, WANs), it is unlikely that the world will settle on one global blockchain system operating universally. The emerging picture will most likely consist of "islands" of blockchain systems, whichlike autonomous systems that make-up the Internet-must be "stitched" together in some fashion to make a coherent unity. Similar to packets of messages traversing different paths through the stitched islands of the Internet, blockchain transactions must be free to traverse the stitched islands of blockchain systems. This freedom of transactions to traverse or move across blockchain systems is not only needed to prevent "asset lock-in" to a given platform, but it is crucial from the point of view survivability of these systems as a whole.

Following from the first fundamental goal of the Internet architecture, the lesson learned there was that interoperability is key to survivability. Thus, interoperability is core to the entire value-proposition of blockchain technology. Interoperability across blockchain systems must be a requirement-both at the mechanical level and at the value level-if blockchain systems and 
technologies are to become the fundamental infrastructure of the future global commerce (see Lipton and Pentland, 2018; Lipton et al., 2018).

In this section we discuss the use of the DTCB for the purpose of addressing some of the security challenges pertaining to blockchain gateways - a notion put forward by Hardjono et al. (2019) as a counterpart to routing gateways (e.g., BGP Routers) in the Internet.

Blockchain gateways provides a number of potential benefits, notably in use-cases involving the transferal of assets across different blockchain systems:

- Better control over ledger visibility: The first potential benefit of gateways in the context of blockchain interoperability is to provide manageable control over the visibility (i.e., read access) of data residing on the ledger within a given private/permissioned blockchain system. The visibility of local ledger data is particularly relevant for inter-domain (cross blockchain) transactions, in cases where one or both of the blockchain systems are private and where the ledger data is confidential.

- Trust establishment across distinct blockchain systems: The second potential benefit of gateways in the context of blockchain interoperability is to support the establishment of trust (i.e., technical-trust) across blockchain autonomous systems. This need for inter-domain trust establishment maybe necessary for scenarios involving high value transactions. Although trust establishment is traditionally performed between two devices, there may be use-cases that require multiple gateways in one system to simultaneously establish group-oriented trust with multiple gateways in another blockchain system.

- Peering-points for service contracts: The third potential benefit of gateways in the context of blockchain interoperability is to serve as the peering-points that are recognized (called out) within peering agreements or contracts. Similar to peering agreements between ISPs in Internet routing, new kinds of peering agreements will need to be developed for blockchain system interoperability in order that these independent systems can interconnect in a secure and reliable fashion.

There are several positive benefits of employing the DTCB model for blockchain gateways:

- Provide higher assurance to nodes participating in consensus protocols: Augment the nodes to employ TCB-related technologies to allow them not only to operate in a provable trustworthy manner but also to allow them to convey this trust in some meaningful way to external entities (e.g., to other peer nodes, and to wallet systems at the end-users).

- Provide foundations for TCB-capable nodes to dynamically become Gateways: In order for distinct blockchain systems to interoperate with each other to achieve service scale (e.g., for upper layer applications), some (or all) nodes in one blockchain system must be able to act as gateways to interact with their corresponding gateways in a different blockchain system.
- Trust establishment across different blockchain systems using ROT: Employ the properties P1 to P4, DP1 and DP2 (see section 4.1) to establish trust between two peer gateways, and between two groups of gateways (multi-gateways).

Similar to IP packet routing autonomous systems, a blockchain autonomous system may consists of numerous nodes that make up the P2P network (Figure 8A). Some of these nodes we designate as gateways for the purpose of blockchain interoperability (Figure 8B). Independent of whether the blockchain is permissionless or permissioned, a number of nodes (or all of the nodes) in the P2P network must have the capability to handle transactions that involve foreign blockchain systems.

Ideally, a node could be a "member" of two (or more) blockchain autonomous systems. In this case, the node would act as a "bridge" between the two blockchain autonomous systems (see Figure 8C). Although this bridge model has its own security challenges, in general we cannot assume that such a node will exist for all blockchain autonomous systems configurations.

As such, we assume here that the most realistic deployment configuration-considering private/permissioned blockchainsis for two gateways to interact, where each gateway represents its "home" blockchain system. This is akin to two BGP routers in the Internet belonging to two ISPs respectively, where the $\mathrm{BGP}$ routers are peered in order for them to exchange route advertisements. Figure 8D illustrates the situation in which two nodes G1 and G2 are acting as gateways between two corresponding blockchain autonomous systems BC1 and BC2.

\subsection{Blockchain Autonomous Systems}

Similar to a routing autonomous system (routing AS) being composed of one or more routing domains, we propose viewing a blockchain system as an autonomous system (blockchain AS).

Thus, just as routers in a routing-domain operate one or more routing protocols to achieve best routes through that domain, nodes in a blockchain AS contribute to maintaining a shared ledger by running one or more ledger management protocols (e.g., consensus algorithms, membership management) to achieve stability and fast convergence (i.e., confirmation throughput) of the ledger in that AS. The division also maps readily into permissioned and permissionless/public blockchains, where each type of community (and each instance) could be viewed as a separate blockchain AS.

Nodes could therefore be classified from the perspective of ledger management as operating either intra-domain or interdomain (across autonomous systems):

- Intra-domain nodes: These are nodes and other entities whose main task is maintaining ledger information and conducting transactions within one blockchain AS. Examples includes nodes which participate in consensus computations (e.g., full mining nodes in Bitcoin), nodes that "orchestrate" consensus computations (e.g., Orderers and Endorsers in Hyperledger Fabric, see Androulaki et al., 2018), and nodes which perform validations only (e.g., Validators in Ripple, see Schwartz et al., 2014).

- Inter-domain gateways: These are nodes and other entities whose main task is dealing with transactions involving 
A

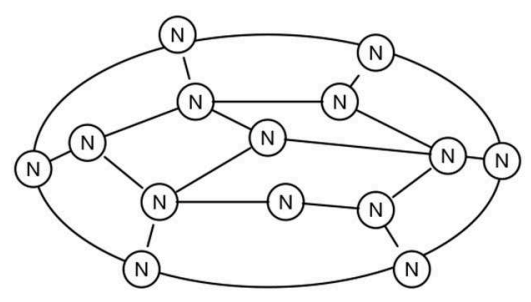

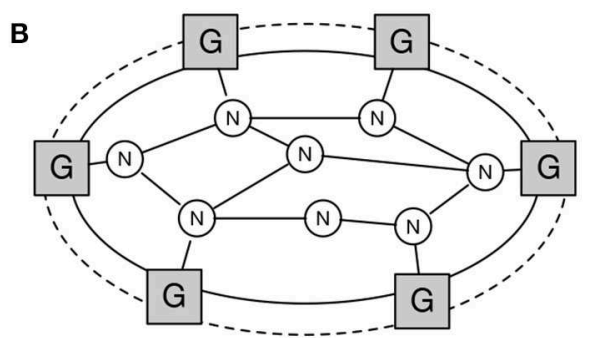

C

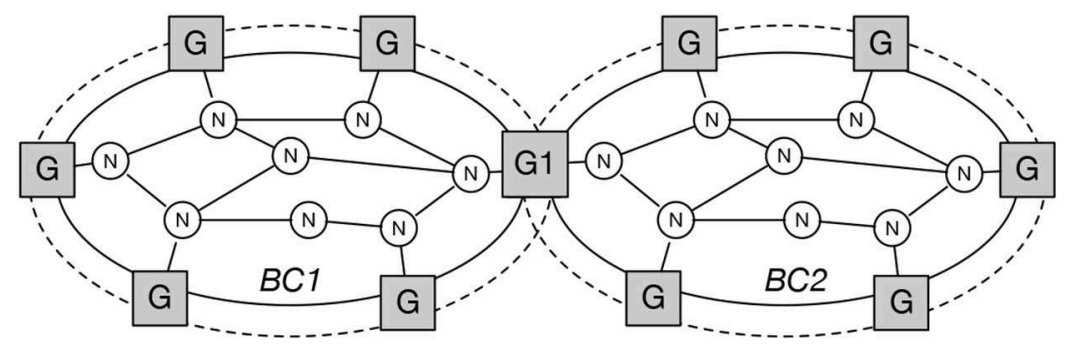

D

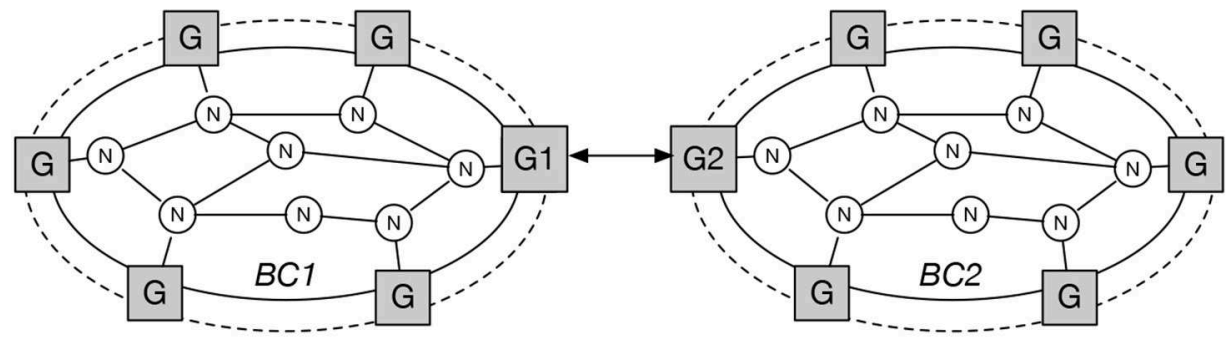

G Gateways (N) Nodes

FIGURE 8 | Illustration of (A) blockchain nodes in P2P network; (B) Some nodes as Gateways; (C) Node G1 acting as a Bridge; (D) Nodes G1 and G2 as Gateways.

different blockchain autonomous systems. We refer to these nodes as inter-domain gateways (or simply "gateways").

Nodes which are gateways must implement very stringent security requirements because they interact with other gateways belonging to different blockchain autonomous systems, and thus different administrative jurisdictions, and potentially different legal jurisdictions.

\subsection{Gateways Between Blockchain Systems}

To illustrate, we sketch a simple example shown in Figure 9 in which an asset recorded in the shared ledger in $\mathrm{BC} 1$ is to be transferred to blockchain system BC2. In Figure 9, a user U1 with Application A has his or her asset ownership (e.g., land title deed) recorded on the shared-ledger inside blockchain $\mathrm{BC} 1$. The user U1 wishes to transfer legal ownership of the asset to a different user $\mathrm{U} 2$ running Application $\mathrm{B}$, and to have the asset recoded authoritatively on the shared-ledger inside blockchain $\mathrm{BC} 2$. We assume both $\mathrm{BC} 1$ and $\mathrm{BC} 2$ are private/ permissioned systems.

In this scenario, the set of gateways in blockchain system $\mathrm{BC} 1$ have agreed to allow G1 to "speak on behalf" of BC1. That is, they have delegated authority to a single gateway G1. Similarly, G2 has been delegated authority to speak on behalf of blockchain system BC2.

The sketch is as follows (Figure 9):

1. The user U1 of Application A initiates the transfer to user U2 running Application B. This consists of the user U1 transmitting a new (inter-domain) transaction within blockchain system $\mathrm{BC} 1$, addressed to user $\mathrm{U} 2$ whose identity (public-key) is present in BC2. Since the destination of this transaction is a public-key located outside BC1 (e.g., $\left.B C 2 / P u b K e y_{U 2}\right)$, this transaction can only be processed by nodes in $\mathrm{BC} 1$ that have the capability of being a gateway (namely G1).

2. Gateway G1 notices the pending (unprocessed) transaction destined for a foreign blockchain BC2. Gateway G1 begins trust establishment with gateway G2 in blockchain system BC2. Because blockchain system BC1 is a private/permissioned system, data in its ledger is not visible from outside the blockchain. As such, gateway G1 has to create a new public transaction-identifier TxID1 for the asset that masks the original transaction-identifier recorded on the private shared ledger in BC1. That is, G1 has to "mask" the original transaction-identifier. 


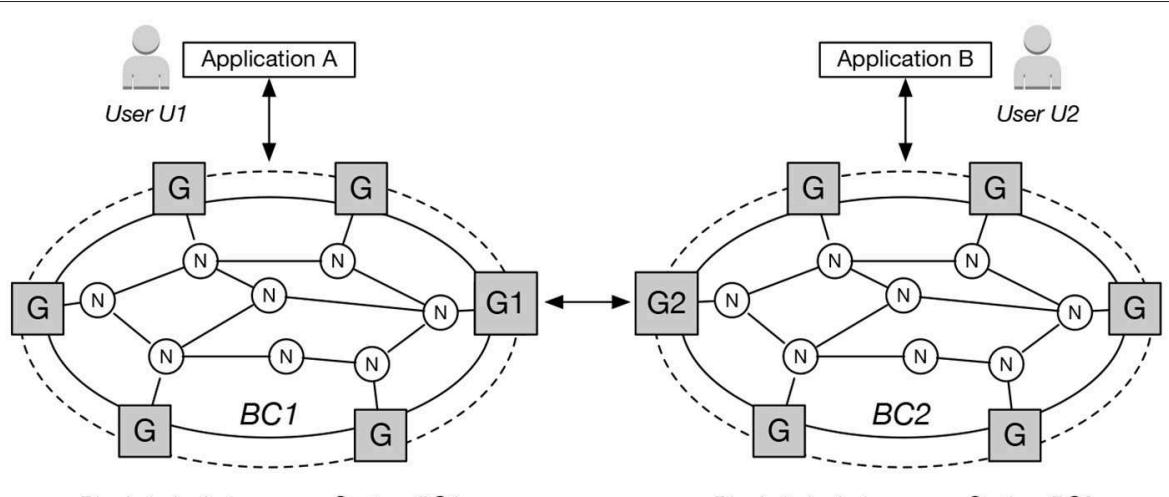

Blockchain Autonomous System BC1

Blockchain Autonomous System BC2

G Gateways (N) Nodes

FIGURE 9 | Transactions crossing blockchain autonomous systems.

3. After pairwise technical-trust has been established between gateways G1 and G2, the gateway G2 proceeds to introduce a new registration-transaction for the asset into the local ledger of blockchain systems BC2. This act is essentially "registering" the soon-to-arrive asset in the shared ledger of BC2. This asset is "locked" (e.g., using 2-Phase Commit or similar construct) by $\mathrm{G} 2$ until G1 settles the transaction in $\mathrm{BC} 1$. While this asset is in locked status in BC2, user U2 is prevented from making use of the asset.

4. After a confirmation has been achieved in the ledger of BC2, the gateway G2 performs two things. First, it creates new public transaction-identifier TxID2 that masks the private transaction-identifier of the asset in BC2; (ii) Secondly, G2 issues a signed assertion to G1 to the effect that the asset labeled TxID2 has been recognized and temporarily registered in the ledger of BC2.

5. Upon receiving the signed assertion from G2, the gateway G1 proceeds to introduce an "invalidation" transaction into the ledger of BC1. In effect, this invalidation transaction marks in the ledger in $\mathrm{BC} 1$ that the asset no longer resides in $\mathrm{BC} 1$. The invalidation transaction in BC1 records both TxID1 and TxID2 for future redirections. This allows for future queriers looking for TxID1 to be redirected to BC2 to obtain TxID2.

6. After the invalidation-transaction is confirmed on the ledger of $\mathrm{BC} 1$, the gateway $\mathrm{G} 1$ issues a signed assertion (to G2) stating that local invalidation-transaction has been confirmed on BC1. The signed assertion uses the TxID1 and TxID2 identifiers, which are public transaction identifiers (not the private identifiers inside $\mathrm{BC} 1$ and $\mathrm{BC} 2$, respectively).

7. Upon receiving the signed assertion from $\mathrm{G} 1$, the gateway $\mathrm{G} 2$ releases the lock on the asset in the ledger of $\mathrm{BC} 1$, thereby allowing its new owner U2 to use the asset.

Several variations of the above example can be devised, improving the efficiency of the messages and transaction throughput. The goal of this example, however, is to illustrate (i) the crucial role that gateways G1 and G2 play in cross-AS transactions; (ii) the relevance of the DTCB model in securing G1 and G2; and (iii) the potential use of the DTCB model

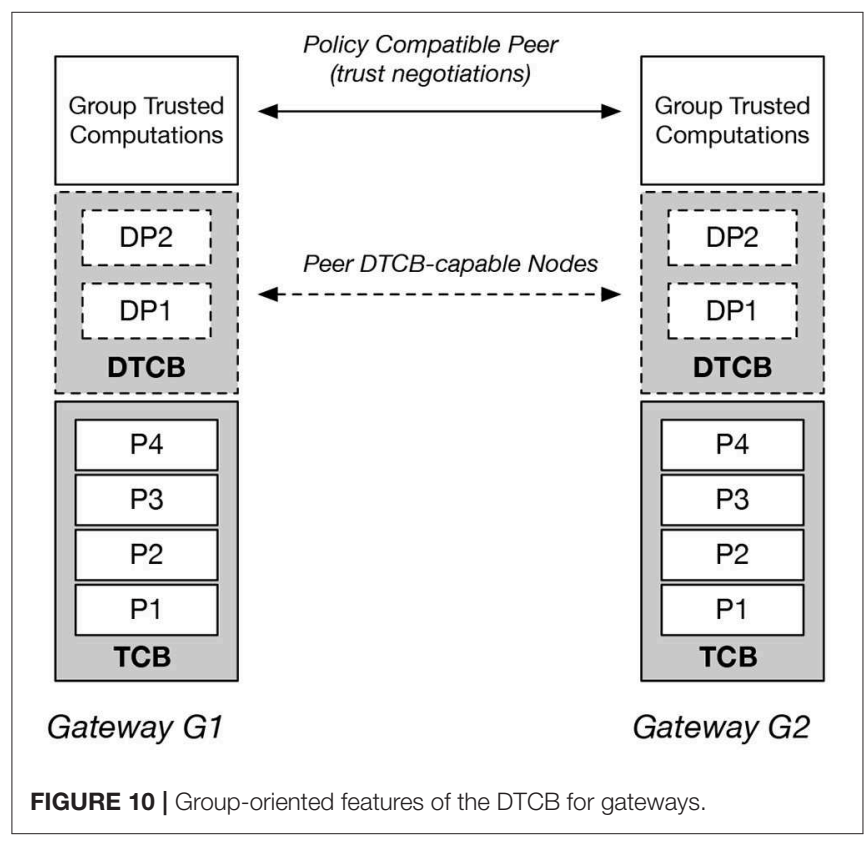

in supporting group-oriented computations that makes use of several gateways in $\mathrm{BC} 1$ simultaneously for increased resiliency (in contrast to the example using one gateway G1 only).

\subsection{Applications of Features of the DTCB for Gateways and Multi-Gateways}

In section 4.1, we discussed a number of properties that support the decentralized TCB model. In this section we briefly review the application of those properties to the blockchain gateways use-case.

As mentioned above, gateways in Internet routing play an important role for connecting two or more routing autonomous systems, allowing both route-advertisements and datagrams to flow through the network. Blockchain gateways, however, have the additional task of acting on behalf of 
its blockchain autonomous system in cases of value-carrying transaction that are inter-domain. This notion of "acting on behalf" of a blockchain autonomous system introduces several interesting challenges in both permissionless and permissioned blockchains (Figure 10):

- Proving DTCB properties by a Gateway locally: In scenarios where the P2P network of nodes consists of a mix of DTCBcapable nodes and non-DTCB nodes, the ability to make use of truthful attestations (Property DP2) allows DTCBcapable nodes to be distinguished (from non-DTCB nodes) as potential gateways.

- Dynamic establishment of Multi-Gateways: A given blockchain autonomous system may operate on the basis of the identification and selection of DTCB-capable nodes as a group gateways or multi-gateways. A multi-gateway is a group of DTCB-capable gateways that must act in unity. All actions to be taken by any member of the group must be based on some group-computation.

- Matching DTCB properties between gateways in different blockchain autonomous systems: As part of technical-trust negotiation and establishment between gateways G1 in BC1 and gateway G2 in BC2 (see Figure 9), G1 and G2 must validate each other's DTCB properties. This may include mutually validating all of the roots of trust in properties P1, $\mathrm{P} 2$, and P3 in each gateway.

- Proving execution of consensus algorithm under shielded processing: The property P2 (see section 4.1) allows DTCBcapable nodes to (i) safely execute consensus algorithms (and other related computations), and (ii) to prove that these algorithms were executed under shielding.

- Majority gateway consensus for sensitive transactions: The set of DTCB-capable gateways may collectively implement governance rules that require sensitive interdomain transactions to be "approved by" the majority of these gateways. That is, gateways may become a subcommunity of nodes, whose majority quorum is needed for inter-domain transactions.

- Proving membership to gateways subcommunity: Depending on the specific implementation of properties P3 and DP1 (see section 4.1), a gateway can prove (a) that it is a DTCB-capable node and (b) that it belongs to the gateways subcommunity. This feature maybe useful in the first stages of gateway-togateway trust negotiations, where an honest gateway is not able to know if its opposite gateway is truly DTCB-capable and that is has been authorized to act as a gateway by virtue of being a member of the subcommunity.

- Gateway initial anonymity to external parties: Being a fully fledged node on the blockchain network, a gateway must always be in a reachable state either through a well-known IP connection or through its public-key which can be found on the blockchain ledger. However, in some cases nodes may wish to initially hide the fact that it is DTCBcapable and appear to be a plain non-DTCB node. This feature may help in reducing possible DDOS attacks on gateways who are members of the gateways-subcommunity. In such DDOS attacks the goal of the attacker (i.e., fake non-DTCB machines) may simply be to exhaust the resources of legitimate gateways through opening numerous fake handshakes.

- DTCB manifest as condition for peering agreements: For peering between two permissioned/private blockchain autonomous systems, a manifest of the list of the minimal set of hardware and software for DTCBcapable nodes provides a way for organizations to build Service Level Agreements (SLAs) based on measurable technical-trust. Property DP2 ensures that a DTCB-capable node can truthfully report its manifest to an external entity as part of satisfying the peering agreement.

- DTCB-assisted multi-party computation: A set of DTCB-capable gateways can collectively use properties P1, P2, and DP2 to jointly perform a given multiparty computation (MPC) (see Lindell, 2003). For example, a given MPC computation maybe designed to yield a common cryptographic key $K_{B C 1, B C 2}$ shared between two opposing groups of gateways (e.g., one group in BC1 and the other in BC2). This would allow any gateways in $\mathrm{BC} 1$ holding key $K_{B C 1, B C 2}$ to begin interacting with any gateway in $\mathrm{BC} 2$ holding the same key.

\subsection{Blockchain Nodes, Gateways, and Privacy}

One issue in using hardware with cryptographic capability, such as the TPM, for implementing some features of blockchain gateways is the potential impact on privacy. For example, in the case of the TPM hardware the key used by a TPM to sign its attestations could be used by an external entity to correlate across different circumstances of usage of these attestations. This in turn could be used to track the platform containing that TPM.

In order to address this potential problem, the Trusted Computing Group (TCG) adopted the Direct Anonymous Attestations (DAA) approach based on the work of Camenisch and Lysyanskaya (2002), Brickell et al. (2004), and Brickell and Li (2012). Generally, the purpose of the DAA scheme is to allow the TPM to issue attestations directly (e.g., to a verifying entity) without the need of a trusted third party, such as a privacysupporting certification authority (called the Privacy CA). The goal is also to prevent the correlation or linking of keys across different usage instances. The DAA was thus included in the TPM v1.2 specifications, and it has been carried over more recently into the TPM v2.0 specifications. Efforts are continuing to improve the DAA capabilities of the TPM (e.g., see Camenisch et al., 2016).

\section{CONCLUSION AND FURTHER CONSIDERATIONS}

Although there has been significant interest and media attention given to the area of blockchain technology, there remains a number of open issues that needs to be addressed. These range from the problem of the concentration of hash-power, 
anonymity of entities, lack of good key management, to the lack of measurable technical-trust required for business agreements and SLAs. As such, there is a strong need for these issues to be addressed before the blockchain technology can become the basis for the future global financial infrastructure.

We believe that a decentralized TCB model is the appropriate technological foundation for providing technical-trust for the blockchain infrastructure. This includes the hardening of individual nodes and systems in the blockchain infrastructure, to providing support for secure group-oriented computationsincluding consensus algorithms and multi-party computationsfor nodes that make-up a blockchain system. This paper devoted considerable attention to the virtualized cloud environments because it is likely that much of the future blockchain infrastructure may operate in cloud environments.

Finally, we discussed the role of gateways in blockchain autonomous systems as the modern counterpart of routing autonomous systems. Aside from providing controlled visibility over data recorded in the shared ledger of private/permissioned blockchains, gateways are needed for the interoperability of independent blockchain systems. We believe that the decentralized TCB model provides the basis for developing solutions that support gateways in establishing technical-trust with each other. The ability to express security quality in some measure based on the DTCB allows blockchain infrastructure owners and operators to develop a common legal framework

\section{REFERENCES}

Anati, I., Gueron, S., Johnson, S. P., and Scarlata, V. R. (2013). Innovative Technology for CPU Based Attestation and Sealing. Technical Report, Intel Corporation. Available online at: https://software.intel.com/sites/default/files/ article/413939/hasp-2013-innovative-technology-for-attestation-and-sealing. pdf.

Android (2019). Verifying Hardware-Backed Key Pairs With Key Attestation. Android Developers Guide. Available online at: https://developer.android.com/ training/articles/security-key-attestation.

Androulaki, E., Barger, A., Bortnikov, V., Cachin, C., Christidis, K., De Caro, A., et al. (2018). "Hyperledger fabric: a distributed operating system for permissioned blockchains," in Proceedings of the Thirteenth EuroSys Conference (New York, NY: ACM), 1-30.

Brickell, E., Camenisch, J., and Chen, L. (2004). "Direct anonymous attestation," in Proceedings of the 11th ACM Conference on Computer and Communications Security CCS2004 (Washington, DC: ACM), 132-145.

Brickell, E., and Li, J. (2012). Enhanced privacy ID: a direct anonymous attestation scheme with enhanced revocation capabilities. IEEE Trans. Depend. Secure Comput. 9, 345-360. doi: 10.1109/TDSC.2011.63

Camenisch, J., Drijvers, M., and Lehmann, A. (2016). "Anonymous attestation using the strong diffie hellman assumption revisited," in Trust and Trustworthy Computing - Proceedings of the 9th International Conference TRUST 2016 (LNCS9824), eds M. Franz and P. Papadimitratos (Vienna: Springer), 1-20.

Camenisch, J., and Lysyanskaya, A. (2002). "A signature scheme with efficient protocols," in Security in Communication Networks (SCN2002) (LNCS 2576), eds S. Cimato, G. Persiano, and C. Galdi (Amalfi: Springer), 268-289.

Camenisch, J., and Van Herreweghen, E. (2002). "Design and implementation of the Idemix anonymous credential system," in Proceedings of the 9th ACM Conference on Computer and Communications Security (Washington, DC: ACM), 21-30.

Cerf, V. G., and Khan, R. E. (1974). A protocol for packet network intercommunication. IEEE Trans. Commun. 22, 637-648. for establishing peering connectivity, and therefore scale. This is how the Internet and IP routing evolved over the past three decades, and we believe this is how the blockchain infrastructure will also evolve.

\section{AUTHOR CONTRIBUTIONS}

All authors listed have made a substantial, direct and intellectual contribution to the work, and approved it for publication.

\section{ACKNOWLEDGMENTS}

We thank Prof. Sandy Pentland and Prof. Alexander Lipton at MIT for support and insights into this work. We thank the following for ideas, critiques and support: Anne Kim, Justin Anderson, Michael Casey, Sandro Lera (MIT); Jerry Cuomo, Gari Singh, Mac Devine, Jeb Linton (IBM); Jan Camenisch (Dfinity). We also thank the numerous colleagues at Intel who have made valuable contributions to trusted computing including Frank McKeen, Claire Vishik, David Grawrock, Vinnie Scarlata, Simon Johnson, David M. Wheeler, and Abhilasha Bhargav-Spantzel as well as those who have helped develop our understanding of cloud, edge, and IoT computing including Francesc Guim, Dario Sabella, Mikko Ylinen, and Kapil Sood. We especially want to thank Jennifer E. Koerv for being an advocate and champion of this work.

Clark, D. (1988). "The design philosophy of the DARPA internet protocols," in Proceedings of SIGCOMM '88, ACM Computer Communication Review, Vol. 18 (Stanford, CA), 106-114.

Hardjono, T., Lipton, A., and Pentland, A. (2019). Towards an interoperability architecture blockchain autonomous systems. IEEE Trans. Eng. Manage. (New York, NY: IEEE) 1-12. doi: 10.1109/TEM.2019.2920154

Hardjono, T., and Smith, N. (2006). TCG Infrastructure Working Group Architecture (Part 2) - Integrity Management - Specification Version 1.0 Rev 1.0. TCG Published Specification, Trusted Computing Group. Available online at: http://www.trustedcomputinggroup.org/resources.

Hardjono, T., and Smith, N. (2016). "Cloud-based commissioning of constrained devices using permissioned blockchains," in Proceedings of the Second ACM International Workshop on IoT Privacy, Trust, and Security (IoTPTS 2016) (Xian: ACM), 29-36.

ISO/IEC (2013). Information Technology - Security Techniques - Anonymous Digital Signatures. International Standard ISO/IEC 20008-1; International Organization for Standardization (ISO).

Jones, M. B. (2015). FIDO 2.0: Key Attestation Format. W3C Member Submission, W3C. Available online at: http://www.w3.org/Submission/fidokey-attestation/.

Lindell, Y. (2003). Composition of Secure Multi-Party Protocols: A Comprehensive Study (LNCS 2815). Berlin: Springer-Verlag.

Lipton, A., Hardjono, T., and Pentland, A. (2018). Digital trade coin (DTC): towards a more stable digital currency. J. R. Soc. Open Sci. 5:180155. doi: 10.1098/rsos. 180155

Lipton, A., and Pentland, A. (2018). Breaking the bank. Sci. Am. 318, 26-31. doi: 10.1038/scientificamerican0118-26

McKeen, F., Alexandrovich, I., Anati, I., Caspi, D., Johnson, S., Leslie-Hurd, R., et al. (2016). "Intel software guard extensions (Intel SGX) support for dynamic memory management inside an enclave," in Proc. Workshop on Hardware and Architectural Support for Security and Privacy (HASP) 2016 (Seoul). Available online at: http://caslab.csl.yale.edu/workshops/hasp2016/program.html.

Mckeen, F., Alexandrovich, I., Berenzon, A., Rozas, C., Shafi, H., Shanbhogue, V., et al. (2013). "Innovative instructions and software model for isolated 
execution," in Proc. Second Workshop on Hardware and Architectural Support for Security and Privacy HASP2013 (Tel-Aviv). Available online at: https://sites. google.com/site/haspworkshop2013/workshop-program.

Microsoft (2017). TPM Key Attestation. Microsoft IT Pro Center Report, Microsoft Inc. Available online at: https://docs.microsoft.com/en-us/windows-server/ identity/ad-ds/manage/component-updates/tpm-key-attestation.

Nakamoto, S. (2008). Bitcoin: A Peer-to-Peer Electronic Cash System.

Schwartz, D., Youngs, N., and Britto, A. (2014). The Ripple Protocol Consensus Algorithm. Technical Report, Ripple Inc.

Trusted Computing Group (2003a). TPM Main - Part 1 Design Principles Specification Version 1.2. TCG Published Specification, Trusted Computing Group. Available online at: http://www.trustedcomputinggroup.org/resources/ tpm_main_specification.

Trusted Computing Group (2003b). TPM Main - Specification Version 1.2. TCG Published Specification, Trusted Computing Group. Available online at: http://www.trustedcomputinggroup.org/resources/tpm_main specification.
Trusted Computing Group (2016). Trusted Platform Architecture Hardware Requirements for a Device Identifier Composition Engine. TCG Committee Draft, Trusted Computing Group. Available online at: https://www. trustedcomputinggroup.org.

Conflict of Interest: NS was employed by company Intel Corporation, USA.

The remaining author declares that the research was conducted in the absence of any commercial or financial relationships that could be construed as a potential conflict of interest.

Copyright (c) 2019 Hardjono and Smith. This is an open-access article distributed under the terms of the Creative Commons Attribution License (CC BY). The use, distribution or reproduction in other forums is permitted, provided the original author(s) and the copyright owner(s) are credited and that the original publication in this journal is cited, in accordance with accepted academic practice. No use, distribution or reproduction is permitted which does not comply with these terms. 